\title{
Liver Injury in COVID-19: Caution and Management
}

\author{
Jiasheng Cao ${ }^{a} \quad$ Xiujun Cai ${ }^{a, b} \quad$ Mingyu Chen ${ }^{a-c}$ \\ ${ }^{a}$ Department of General Surgery, Sir Run-Run Shaw Hospital, Zhejiang University, \\ Hangzhou, China; ${ }^{b}$ Engineering Research Center of Cognitive Healthcare of Zhejiang \\ Province, Hangzhou, China; ' Zhejiang University School of Medicine, Hangzhou, China
}

\section{Dear Editor,}

COVID-19 is an emerging infectious disease of SARS-CoV-2 origin that has become a global threat to human health, and the number of confirmed cases has exceeded 3,500,000 worldwide as of May 4, 2020. Previous studies almost focused on lung injury of COVID-19, while few articles have taken liver involvement into consideration, which ranged from 14.8 to $53 \%$ [1].

Liver impairment is partly due to drug hepatotoxicity. Currently, medicines prescribed to treat COVID-19 include oseltamivir, ritonavir/lopinavir, ribavirin, and hydroxychloroquine sulfate, or chloroquine phosphate, which are metabolized in the liver and would produce hepatotoxicity, and the inducible liver injury can impair metabolism and excretion of the medications in turn. The postmortem biopsies of a COVID-19 patient showed mild lobular and portal activity, and moderate microvascular steatosis, which indicated that liver injury was SARS-CoV-2 infection-related or drug-related [2]. Therefore, clinicians should concentrate on liver injury and monitoring liver function in COVID-19 patients.

Liver injury in patients comorbid with chronic liver diseases and COVID-19 should be evaluated due to the global disease burden of chronic liver diseases, including chronic viral hepatitis, non-alcoholic fatty liver disease, liver cirrhosis, liver cancer, and so on. Although the interaction between COVID-19 and existing chronic liver diseases has not been studied, immune dysfunction is exactly a common feature in those patients and might be associated with disease severity and mortality. Moreover, considering the immunocompromised status, intensive surveillance and individually tailored therapy are appropriate for the above patients.

Notably, recipients of liver transplantation may have high risks of being infected with COVID-19 by hospital exposure during the outbreak [3]. Recipients may have higher potential to develop severe immune suppression and postoperative infection, which increase the rate of multisystem organ damage or death. There exists a contradiction that excessive immunosuppression leads to severe infections, while insufficient immunosuppression causes graft loss due to rejection reaction. A case report by Qin et al. [4] showed that a recipient had an 
extended duration of being a virus carrier. Even if the COVID-19 RT-PCR test was negative during the treatment, it may be positive later due to the rise of viral load as the dosage of immunosuppression medicine was increased. Thus, strict screening guidance and prolonged follow-up should be offered for liver recipients with COVID-19 infection.

Above all, clinicians should pay more attention to liver injury in COVID-19 patients and take active measures to help them get through the difficult period.

\section{Acknowledgement}

We thank Jiliang Shen and Xu Feng for their efforts spent in checking the grammar errors in the manuscript.

\section{Disclosure Statement}

The authors have no conflicts of interest to disclose.

\section{Funding Sources}

The authors received no funding support for this study.

\section{Author Contributions}

J.C. and X.C. designed and wrote the manuscript. M.C. supervised the study and revised the manuscript. All authors read and approved the final manuscript.

\section{References}

1 Chen N, Zhou M, Dong X, Qu J, Gong F, Han Y, et al. Epidemiological and clinical characteristics of 99 cases of 2019 novel coronavirus pneumonia in Wuhan, China: a descriptive study. Lancet. 2020 Feb;395(10223):50713.

2 Xu Z, Shi L, Wang Y, Zhang J, Huang L, Zhang C, et al. Pathological findings of COVID-19 associated with acute respiratory distress syndrome. Lancet Respir Med. 2020 Apr;8(4):420-2.

3 Wang D, Hu B, Hu C, Zhu F, Liu X, Zhang J, et al. Clinical Characteristics of 138 Hospitalized Patients With 2019 Novel Coronavirus-Infected Pneumonia in Wuhan, China. JAMA. 2020. Feb;323(11):1061-9.

4 Qin J, Wang H, Qin X, Zhang P, Zhu L, Cai J, et al. Perioperative Presentation of COVID-19 Disease in a Liver Transplant Recipient. Hepatology. 2020 Mar. https://doi.org/10.1002/hep.31257. 